\title{
Lightning strike impacts on hybrid Photovoltaic-Wind system
}

\begin{abstract}
Study the impacts of lightning-induced transient overvoltage on a hybrid PV-Wind system has been addressed in this work. Overvoltage that is generated due to lightning stroke travels along the system where it can be very harmful to the expensive equipment of the system such as PV models, inverters, charge controllers, batteries, transformers, and generator etc. The simulation model of a system has been completed by using PSCAD/EMTDC software. The system comprises of 2 MW PV farm, battery system, 2.1 MW wind farm and loads which are all connected to the common $\mathrm{AC}$ bus and then to the utility grid through an interfacing transformer. Lightning current is generated by using the double exponential function, from the simulation results, when the lightning current is injected to the AC and DC sides of PV system, the transient current and voltage have appeared at different points of the hybrid system. The results were obtained for $8 / 20 \mu$ s and $10 / 350 \mu$ s standards lightning waveforms with current magnitude of $100 \mathrm{kA}$.
\end{abstract}

Keyword: Hybrid PV-Wind system modelling; Lightning; Transient impact; Overvoltage; Overcurrent 